\title{
A Connection Between Score Matching and Denoising Autoencoders
}

\author{
Pascal Vincent \\ vincentp@iro.umontreal.ca \\ Dept. IRO, Université de Montréal, \\ CP 6128, Succ. Centre-Ville, Montréal (QC) H3C 3J7, Canada.
}

Technical Report 1358

Département d'Informatique et de Recherche Opérationnelle

December 2010

\section{THIS IS A PREPRINT VERSION OF A NOTE THAT HAS BEEN ACCEPTED FOR PUBLICATION IN NEURAL COMPUTATION.}

Keywords: autoencoder, energy based models, score matching, denoising, density estimation.

\begin{abstract}
Denoising autoencoders have been previously shown to be competitive alternatives to Restricted Boltzmann Machines for unsupervised pre-training of each layer of a deep architecture. We show that a simple denoising autoencoder training criterion is equivalent to matching the score (with respect to the data) of a specific energy based model to that of a non-parametric Parzen density estimator of the data. This yields several useful insights. It defines a proper probabilistic model for the denoising autoencoder technique which makes it in principle possible to sample from them or to rank examples by their energy. It suggests a different way to apply score matching that is related to learning to denoise and does not require computing second derivatives. It justifies the use of tied weights between the encoder and decoder, and suggests ways to extend the success of denoising autoencoders to a larger family of energy-based models.
\end{abstract}

\section{Introduction}

This note uncovers an unsuspected link between the score matching technique (Hyvärinen, 2005; Hyvärinen, 2008) for learning the parameters of unnormalized density models over continuous-valued data, and the training of denoising autoencoders (Vincent et al., 2008, 2010).

Score matching (SM) is an alternative to the maximum likelihood principle suitable for unnormalized probability density models whose partition function is intractable. Its 
relationship to maximum likelihood has been investigated by Lyu (2009) who formally relates the Fisher divergence that yields score matching and the Kullback-Leibler divergence that yields maximum likelihood. Interestingly, his formal analysis indicates that score matching searches for parameters that are more robust to small-noise perturbations of the training data (Lyu, 2009). Score matching has also been recast as a special case under the more general frameworks of generalized score matching (Lyu, 2009; Marlin et al., 2009) and minimum probability flow (Sohl-Dickstein et al., 2009), allowing generalizations of score matching to discrete distributions (Hyvärinen, 2007b; Lyu, 2009; Sohl-Dickstein et al., 2009). The minimum probability flow paradigm is particularly interesting as it unifies several recent alternative parameter-estimation methods, both for continuous and discrete data, under a single unified view ${ }^{1}$. Recently, Kingma and LeCun (2010) investigated a regularized form of score matching which adds a specific regularization term to the original score matching objective. Its relationship to the present work will be discussed in detail in Section 5.

Denoising Autoencoders (DAE) were proposed by Vincent et al. (2008) as a simple and competitive alternative to the Contrastive-Divergence-trained Restricted Boltzmann Machines (RBM) used by Hinton et al. (2006) for pretraining deep networks (Erhan et al., 2010; Vincent et al., 2010). Previous studies have already pointed out connections between SM and Contrastive Divergence (Hyvärinen, 2007a; Sohl-Dickstein et al., 2009), have connected SM to optimal denoising for Gaussian noise with infinitesimal variance (Hyvärinen, 2008) and have shown that training Gaussian Binary RBM with SM is equivalent to training a regular (non-denoising) autoencoder with an additional regularization term (Swersky, 2010). The present study however is the first to recast the training of a DAE as a form of regularized score matching. This connection yields insights relevant to both research directions and suggests a novel parameter estimation technique that has its roots in both DAE and SM.

We begin by a very brief presentation of the DAE architecture for continuous-valued inputs in Section 2 and of the SM technique in Section 3. This allows us to introduce our formalism and precise terminology. Then, in section 4, we connect the denoising autoencoder objective to score matching. We conclude by a discussion on how our findings advance our understanding of both approaches.

\section{Notation}

We are interested in techniques that learn the parameters $\theta$ of a model by minimizing some objective function $J(\theta)$. For uniformity of notation, all distributions will be represented by their probability density functions (pdf) on $\mathbb{R}^{d}$. The pdf for discrete distributions will be expressed with Dirac-deltas $\delta$.

$$
\begin{aligned}
q(\mathbf{x}) & \text { Unknown true pdf. } \mathbf{x} \in \mathbb{R}^{d} . \\
D_{n}=\left\{\mathbf{x}^{(1)}, \ldots, \mathbf{x}^{(n)}\right\} & \text { Training set: i.i.d. sample from } q .
\end{aligned}
$$

\footnotetext{
${ }^{1}$ Specifically score matching (Hyvärinen, 2005), minimum velocity learning (Movellan, 2008), and certain forms of contrastive divergence (Hinton, 2002; Welling and Hinton, 2002) are all recast as minimizing the Kullback-Leibler divergence between the data distribution and the distribution obtained after running, for infinitesimal time, a dynamic that would transform it into the model distribution (SohlDickstein et al., 2009).
} 


$$
\begin{aligned}
q_{0}(\mathbf{x})=\frac{1}{n} \sum_{i=1}^{n} \delta\left(\left\|\mathbf{x}-\mathbf{x}^{(i)}\right\|\right) & \text { Empirical pdf associated with } D_{n} . \\
q_{\sigma}(\tilde{\mathbf{x}} \mid \mathbf{x})=\frac{1}{(2 \pi)^{d / 2} \sigma^{d}} e^{-\frac{1}{2 \sigma^{2}}\|\tilde{\mathbf{x}}-\mathbf{x}\|^{2}} & \text { Smoothing kernel or noise model: isotropic } \\
& \text { Gaussian of variance } \sigma^{2} . \\
q_{\sigma}(\tilde{\mathbf{x}}, \mathbf{x})=q_{\sigma}(\tilde{\mathbf{x}} \mid \mathbf{x}) q_{0}(\mathbf{x}) & \text { Joint pdf. } \\
q_{\sigma}(\tilde{\mathbf{x}})=\frac{1}{n} \sum_{t=1}^{n} q_{\sigma}\left(\tilde{\mathbf{x}} \mid \mathbf{x}^{(t)}\right) & \text { Parzen density estimate based on } D_{n} \\
& \text { obtainable by marginalizing } q_{\sigma}(\tilde{\mathbf{x}}, \mathbf{x}) . \\
p(\mathbf{x} ; \theta) & \text { Density model with parameters } \theta . \\
J_{1} \smile J_{2} & \text { Means } J_{1}(\theta) \text { and } J_{2}(\theta) \text { are equivalent } \\
& \text { optimization objectives }{ }^{2} . \\
\mathbb{E}_{q(\mathbf{x})[g(\mathbf{x})]=\int_{\mathbf{x}} q(\mathbf{x}) g(\mathbf{x}) d \mathbf{x}} & \text { Expectation with respect to distribution } q . \\
\langle\mathbf{u}, \mathbf{v}\rangle=\sum_{i} \mathbf{u}_{i} \mathbf{v}_{i} & \text { Dot product between two vectors. } \\
\|\mathbf{u}\|=\sqrt{\langle\mathbf{u}, \mathbf{u}\rangle} & \text { Euclidean norm of vector u. } \\
\operatorname{softplus}(x)=\log \left(1+e^{x}\right) & \text { Will be applied elementwise to vectors. } \\
\operatorname{sigmoid}(x)=\frac{1}{1+e^{-x}}=\operatorname{softplus}(x) & \text { Will be applied elementwise to vectors. } \\
\mathbf{I} & \text { Identity matrix. } \\
\mathbf{W}^{T} & \text { Transpose of matrix } \mathbf{W} . \\
\mathbf{W}_{i} & \text { Vector for } i^{t h} \text { row of } \mathbf{W} . \\
\mathbf{W}_{\cdot, j} & \text { Vector for } j^{t h} \text { column of W. }
\end{aligned}
$$

\section{Denoising Autoencoders}

Denoising Autoencoders (DAEs) are a simple modification of classical autoencoder neural networks that are trained, not to reconstruct their input, but rather to denoise an artificially corrupted version of their input (Vincent et al., 2008, 2010). Whereas an over-complete regular autoencoder can easily learn a useless identity mapping, a DAE must extract more useful features in order to solve the much harder denoising problem. DAEs have proven to be an empirically successful alternative to Restricted Boltzmann Machines (RBM) for pre-training deep networks (Vincent et al., 2008, 2010; Erhan et al., 2010). Denoising autoencoders have also been used in different contexts in the earlier works of LeCun (1987); Gallinari et al. (1987); Seung (1998).

In this study, we will consider the denoising version of a simple classical autoencoder that uses a single sigmoidal hidden layer. Since data points originate from a continuous real valued distribution it is natural to use a linear decoder with a squared reconstruction loss ${ }^{3}$. We will be using tied weights whereby encoder and decoder share the same linear transformation parameters. The considered corruption is additive isotropic Gaussian noise. A detailed description of the architecture follows:

- A training input $\mathrm{x} \in D_{n}$ is first corrupted by additive Gaussian noise of covariance $\sigma^{2} \mathbf{I}$ yielding corrupted input $\tilde{\mathbf{x}}=\mathbf{x}+\epsilon, \epsilon \sim \mathcal{N}\left(0, \sigma^{2} \mathbf{I}\right)$. This corresponds to

\footnotetext{
${ }^{2}$ Equivalence will be asserted when $J_{2}=\alpha J_{1}+\beta$ with $\alpha>0, \beta \in \mathbb{R}$. Indeed a gradient-based optimization algorithm, when starting from some initial $\theta$ value, should land in the exact same minimum whether optimizing $J_{1}$ or $J_{2}$ (this may however require learning rate adjustment to compensate for scaling factor $\alpha$ ).

${ }^{3}$ as opposed to a linear+sigmoid decoder with a Bernoulli cross-entropy loss, which would be the preferred choice for binary input.
} 
conditional density $q_{\sigma}(\tilde{\mathbf{x}} \mid \mathbf{x})=\frac{1}{(2 \pi)^{d / 2} \sigma^{d}} e^{-\frac{1}{2 \sigma^{2}}\|\tilde{\mathbf{x}}-\mathbf{x}\|^{2}}$.

- The corrupted version $\tilde{\mathbf{x}}$ is encoded into a hidden representation $\mathbf{h} \in \mathbb{R}^{d_{h}}$ through an affine mapping followed by a nonlinearity:

$\mathbf{h}=\operatorname{encode}(\tilde{\mathbf{x}})=\operatorname{sigmoid}(\mathbf{W} \tilde{\mathbf{x}}+\mathbf{b})$, where $\tilde{\mathbf{x}} \in \mathbb{R}^{d}, \mathbf{h} \in(0,1)^{d_{h}}, \mathbf{W}$ is a $d_{h} \times d$ matrix and $\mathbf{b} \in \mathbb{R}^{d_{h}}$.

- The hidden representation $\mathbf{h}$ is decoded into reconstruction $\mathbf{x}^{\mathbf{r}}$ through affine mapping: $\mathbf{x}^{\mathbf{r}}=\operatorname{decode}(\mathbf{h})=\mathbf{W}^{T} \mathbf{h}+\mathbf{c}$, where $\mathbf{c} \in \mathbb{R}^{d}$.

- The parameters $\theta=\{\mathbf{W}, \mathbf{b}, \mathbf{c}\}$ are optimized so that the expected squared reconstruction error $\left\|\mathbf{x}^{\mathbf{r}}-\mathbf{x}\right\|^{2}$ is minimized, i.e. the objective function being minimized by such a denoising autoencoder (DAE) is:

$$
\begin{aligned}
J_{D A E \sigma}(\theta) & =\mathbb{E}_{q_{\sigma}(\tilde{\mathbf{x}}, \mathbf{x})}\left[\|\operatorname{decode}(\operatorname{encode}(\tilde{\mathbf{x}}))-\mathbf{x}\|^{2}\right] \\
& \left.=\mathbb{E}_{q_{\sigma}(\tilde{\mathbf{x}}, \mathbf{x})}\left[\| \mathbf{W}^{T} \operatorname{sigmoid}(\mathbf{W} \tilde{\mathbf{x}}+\mathbf{b})\right)+\mathbf{c}-\mathbf{x} \|^{2}\right] .
\end{aligned}
$$

\section{Score Matching}

\subsection{Explicit Score Matching (ESM)}

Score Matching was introduced by Hyvärinen (2005) as a technique to learn the parameters $\theta$ of probability density models $p(\mathbf{x} ; \theta)$ with intractable partition function $Z(\theta)$, where $p$ can be written as

$$
p(\mathbf{x} ; \theta)=\frac{1}{Z(\theta)} \exp (-E(\mathbf{x} ; \theta))
$$

$E$ is called the energy function. Following Hyvärinen (2005), we will call score the gradient of the $\log$ density with respect to the data vector: $\psi(\mathbf{x} ; \theta)=\frac{\partial \log p(\mathbf{x} ; \theta)}{\partial \mathbf{x}}$. Beware that this usage differs slightly from traditional statistics terminology where score usually refers to the derivative of the log likelihood with respect to parameters, whereas here we are talking about a score with respect to the data. The core principle of score matching (Hyvärinen, 2005) is to learn $\theta$ so that $\psi(\mathbf{x} ; \theta)=\frac{\partial \log p(\mathbf{x} ; \theta)}{\partial \mathbf{x}}$ best matches the corresponding score of the true distribution, i.e. $\frac{\partial \log q(\mathbf{x})}{\partial x}$. The corresponding objective function to be minimized is the expected squared error between these two vectors, i.e.

$$
J_{E S M q}(\theta)=\mathbb{E}_{q(\mathbf{x})}\left[\frac{1}{2}\left\|\psi(\mathbf{x} ; \theta)-\frac{\partial \log q(\mathbf{x})}{\partial \mathbf{x}}\right\|^{2}\right] .
$$

We refer to this formulation as explicit score matching (ESM).

Note that the score $\psi(\mathbf{x} ; \theta)$ does not depend on troublesome $Z(\theta)$. But since $q$ is unknown, we do not have explicit regression targets $\frac{\partial \log q(\mathbf{x})}{\partial \mathbf{x}}$. Hyvärinen (2005) mentions in passing that non-parametric methods might be used to estimate those, and we shall later pay closer attention to this possibility. 


\subsection{Implicit Score Matching (ISM)}

Hyvärinen (2005) instead proceeds by proving the following remarkable property:

$$
\underbrace{\mathbb{E}_{q(\mathbf{x})}\left[\frac{1}{2}\left\|\psi(\mathbf{x} ; \theta)-\frac{\partial \log q(\mathbf{x})}{\partial \mathbf{x}}\right\|^{2}\right]}_{J_{E S M q}(\theta)}=\underbrace{\mathbb{E}_{q(\mathbf{x})}\left[\frac{1}{2}\|\psi(\mathbf{x} ; \theta)\|^{2}+\sum_{i=1}^{d} \frac{\partial \psi_{i}(\mathbf{x} ; \theta)}{\partial \mathbf{x}_{i}}\right]}_{J_{I S M q}(\theta)}+C_{1}
$$

where $\psi_{i}(\mathbf{x} ; \theta)=\psi(\mathbf{x} ; \theta)_{i}=\frac{\partial \log p(\mathbf{x} ; \theta)}{\partial \mathbf{x}_{i}}$, and $C_{1}$ is a constant that does not depend on $\theta$. This yields an implicit score matching objective $J_{I S M q}$ that no longer requires having an explicit score target for $q$ but is nevertheless equivalent to $J_{E S M q}$. Hyvärinen (2005) formally shows that, provided $q(\mathbf{x})$ and $\psi(\mathbf{x} ; \theta)$ satisfy some weak regularity conditions $^{4}$, we have

$$
J_{E S M q} \smile J_{I S M q}
$$

\subsection{Finite Sample Version of Implicit Score Matching}

Since we only have samples $D_{n}$ from $q$, Hyvärinen proposes to optimize the finite sample version of $J_{I S M q}$ which, following our notation, we shall write $J_{I S M q_{0}}$ :

$$
\begin{aligned}
J_{I S M q_{0}}(\theta) & =\mathbb{E}_{q_{0}(\mathbf{x})}\left[\frac{1}{2}\|\psi(\mathbf{x} ; \theta)\|^{2}+\sum_{i=1}^{d} \frac{\partial \psi_{i}(\mathbf{x} ; \theta)}{\partial \mathbf{x}_{i}}\right] \\
& =\frac{1}{n} \sum_{t=1}^{n}\left(\frac{1}{2}\left\|\psi\left(\mathbf{x}^{(t)} ; \theta\right)\right\|^{2}+\sum_{i=1}^{d} \frac{\partial \psi_{i}\left(\mathbf{x}^{(t)} ; \theta\right)}{\partial \mathbf{x}_{i}}\right) .
\end{aligned}
$$

$J_{I S M q_{0}}$ is asymptotically equivalent to $J_{I S M q}$ when $n \rightarrow \infty$ and hence asymptotically equivalent to objective $J_{E S M q}$. This can be summarized as

$$
J_{E S M q} \smile J_{I S M q} \smile \lim _{n \rightarrow \infty} J_{I S M q_{0}} .
$$

What happens in the transition from $J_{I S M q}$ to finite sample version $J_{I S M q_{0}}$ is however not entirely clear. Concerns regarding the stability of the resulting criterion were raised by Kingma and LeCun (2010), who propose instead to optimize a regularized version of $J_{I S M q_{0}}$ :

$$
J_{\text {ISMreg }}(\theta)=J_{I S M q_{0}}(\theta)+\lambda \sum_{i=1}^{d}\left(\frac{\partial \psi_{i}\left(\mathbf{x}^{(t)} ; \theta\right)}{\partial \mathbf{x}_{i}}\right)^{2},
$$

where the strength of the additional regularization term is controlled by hyperparameter $\lambda$. The relationship between this criterion and the criteria we propose below will be further discussed in Section 5.

\footnotetext{
${ }^{4}$ Namely: $q(\mathbf{x})$ and $\psi(\mathbf{x} ; \theta)$ are differentiable, $\mathbb{E}_{q(\mathbf{x})}\left[\left\|\frac{\partial \log q(\mathbf{x})}{\partial \mathbf{x}}\right\|^{2}\right]$ is finite, and for any $\theta$, $\mathbb{E}_{q(\mathbf{x})}\left[\|\psi(\mathbf{x} ; \theta)\|^{2}\right]$ is finite and $\lim _{\|\mathbf{x}\| \rightarrow \infty} q(\mathbf{x}) \psi(\mathbf{x} ; \theta)=0$.
} 


\section{Linking Score Matching to the Denoising Autoencoder Objective}

\subsection{Matching the Score of a Non-Parametric Estimator}

As previously stated, the possibility of matching the score $\psi(\mathbf{x} ; \theta)$ with an explicit target score for $q$ obtained through non-parametric estimation was mentioned but not pursued in Hyvärinen (2005). We now examine this possibility more closely. Explicitly matching $\psi(\mathbf{x} ; \theta)$ with the score of Parzen windows density estimator $q_{\sigma}(\tilde{\mathbf{x}})$ yields the following objective:

$$
J_{E S M q_{\sigma}}(\theta)=\mathbb{E}_{q_{\sigma}(\tilde{\mathbf{x}})}\left[\frac{1}{2}\left\|\psi(\tilde{\mathbf{x}} ; \theta)-\frac{\partial \log q_{\sigma}(\tilde{\mathbf{x}})}{\partial \tilde{\mathbf{x}}}\right\|^{2}\right] .
$$

For $\sigma>0, q_{\sigma}$ is differentiable, decreases to 0 at infinity, and $\mathbb{E}_{q_{\sigma}(\tilde{\mathbf{x}})}\left[\left\|\frac{\partial \log q_{\sigma}(\tilde{\mathbf{x}})}{\partial \tilde{\mathbf{x}}}\right\|^{2}\right]$ is finite. All regularity conditions are satisfied, so the same equivalence with ISM as in Eq. 3 holds, i.e.

$$
J_{E S M q_{\sigma}} \smile J_{I S M q_{\sigma}} .
$$

Note that this equivalence however breaks in the limit $\sigma \rightarrow 0$, because $q_{\sigma}$ no longer satisfies these regularity conditions, and $J_{E S M q \sigma}$ can no longer be computed (whereas $J_{I S M q_{\sigma}}$ remains well-behaved).

\subsection{Denoising Score Matching (DSM)}

Let us now consider a slightly different objective, that is inspired by both the Score Matching principle and by the Denoising Autoencoder approach of using pairs of clean and corrupted examples $(\mathbf{x}, \tilde{\mathbf{x}})$. For joint density $q_{\sigma}(\tilde{\mathbf{x}}, \mathbf{x})=q_{\sigma}(\tilde{\mathbf{x}} \mid \mathbf{x}) q_{0}(\mathbf{x})$, we define the following denoising score matching (DSM) objective:

$$
J_{D S M q_{\sigma}}(\theta)=\mathbb{E}_{q_{\sigma}(\mathbf{x}, \tilde{\mathbf{x}})}\left[\frac{1}{2}\left\|\psi(\tilde{\mathbf{x}} ; \theta)-\frac{\partial \log q_{\sigma}(\tilde{\mathbf{x}} \mid \mathbf{x})}{\partial \tilde{\mathbf{x}}}\right\|^{2}\right] .
$$

The underlying intuition is that following the gradient $\psi$ of the log density at some corrupted point $\tilde{\mathbf{x}}$ should ideally move us towards the clean sample $\mathbf{x}$. Note that with the considered Gaussian kernel we have

$$
\frac{\partial \log q_{\sigma}(\tilde{\mathbf{x}} \mid \mathbf{x})}{\partial \tilde{\mathbf{x}}}=\frac{1}{\sigma^{2}}(\mathbf{x}-\tilde{\mathbf{x}}) .
$$

Direction $\frac{1}{\sigma^{2}}(\mathbf{x}-\tilde{\mathbf{x}})$ clearly corresponds to moving from $\tilde{\mathbf{x}}$ back towards clean sample $\mathbf{x}$, and we want $\psi$ to match that as best it can.

Now this alternate objective, inspired by denoising autoencoders, is equivalent to explicit score matching. Formally,

$$
J_{E S M q_{\sigma}} \smile J_{D S M q_{\sigma}}
$$

The proof is in the appendix, and does not depend on the particular form of $q_{\sigma}(\tilde{\mathbf{x}} \mid \mathbf{x})$ or $q(\mathbf{x})$ as long as $\log q_{\sigma}(\tilde{\mathbf{x}} \mid \mathbf{x})$ is differentiable with respect to $\tilde{\mathbf{x}}$. 


\subsection{An Energy Function that Yields the Denoising Autoencoder Ob- jective}

Let us now choose for model $p$ the form

$$
\begin{aligned}
p(\mathbf{x} ; \theta) & =\frac{1}{Z(\theta)} \exp (-E(x ; \theta)) \\
E(\mathbf{x} ; \underbrace{\mathbf{W}, \mathbf{b}, \mathbf{c}}_{\theta}) & =-\frac{\langle\mathbf{c}, \mathbf{x}\rangle-\frac{1}{2}\|\mathbf{x}\|^{2}+\sum_{j=1}^{d_{h}} \operatorname{softplus}\left(\left\langle\mathbf{W}_{j}, \mathbf{x}\right\rangle+\mathbf{b}_{j}\right)}{\sigma^{2}} .
\end{aligned}
$$

We then have

$$
\begin{aligned}
\psi_{i}(\mathbf{x} ; \theta) & =\frac{\partial \log p(\mathbf{x} ; \theta)}{\partial \mathbf{x}_{i}} \\
& =-\frac{\partial E}{\partial \mathbf{x}_{i}} \\
& =\frac{1}{\sigma^{2}}\left(\mathbf{c}_{i}-\mathbf{x}_{i}+\sum_{j=1}^{d_{h}} \operatorname{softplus}^{\prime}\left(\left\langle\mathbf{W}_{j}, \mathbf{x}\right\rangle+\mathbf{b}_{j}\right) \frac{\partial\left(\left\langle\mathbf{W}_{j}, \mathbf{x}\right\rangle+\mathbf{b}_{j}\right)}{\partial \mathbf{x}_{i}}\right) \\
& =\frac{1}{\sigma^{2}}\left(\mathbf{c}_{i}-\mathbf{x}_{i}+\sum_{j=1}^{d_{h}} \operatorname{sigmoid}\left(\left\langle\mathbf{W}_{j}, \mathbf{x}\right\rangle+\mathbf{b}_{j}\right) \mathbf{W}_{j i}\right) \\
& =\frac{1}{\sigma^{2}}\left(\mathbf{c}_{i}-\mathbf{x}_{i}+\left\langle\mathbf{W}_{\cdot i}, \operatorname{sigmoid}(\mathbf{W} \mathbf{x}+\mathbf{b})\right\rangle\right),
\end{aligned}
$$

which we can write as the single equation

$$
\psi(\mathbf{x} ; \theta)=\frac{1}{\sigma^{2}}\left(\mathbf{W}^{T} \operatorname{sigmoid}(\mathbf{W} \mathbf{x}+\mathbf{b})+\mathbf{c}-\mathbf{x}\right) .
$$

Substituting Eq. 10 and Eq. 13 in the expression for $J_{D S M q_{\sigma}}$ (Eq. 9) we get, for this choice of Parzen kernel and density model,

$$
\begin{aligned}
J_{D S M q_{\sigma}}(\theta) & =\mathbb{E}_{q_{\sigma}(\mathbf{x}, \tilde{\mathbf{x}})}\left[\frac{1}{2}\left\|\psi(\tilde{\mathbf{x}} ; \theta)-\frac{\partial \log q_{\sigma}(\tilde{\mathbf{x}} \mid \mathbf{x})}{\partial \tilde{\mathbf{x}}}\right\|^{2}\right] \\
& =\mathbb{E}_{q_{\sigma}(\mathbf{x}, \tilde{\mathbf{x}})}\left[\frac{1}{2}\left\|\frac{1}{\sigma^{2}}\left(\mathbf{W}^{T} \operatorname{sigmoid}(\mathbf{W} \tilde{\mathbf{x}}+\mathbf{b})+\mathbf{c}-\tilde{\mathbf{x}}\right)-\frac{1}{\sigma^{2}}(\mathbf{x}-\tilde{\mathbf{x}})\right\|^{2}\right] \\
& =\frac{1}{2 \sigma^{4}} \mathbb{E}_{q_{\sigma}(\mathbf{x}, \tilde{\mathbf{x}})}\left[\left\|\mathbf{W}^{T} \operatorname{sigmoid}(\mathbf{W} \tilde{\mathbf{x}}+\mathbf{b})+\mathbf{c}-\mathbf{x}\right\|^{2}\right] \\
& =\frac{1}{2 \sigma^{4}} J_{D A E \sigma}(\theta) .
\end{aligned}
$$

We have thus shown that

$$
J_{D S M q_{\sigma}} \smile J_{D A E \sigma}
$$




\section{Discussion}

Putting together equations 8,11 and 14, we can write, for $\sigma>0$,

$$
J_{I S M q_{\sigma}} \smile J_{E S M q_{\sigma}} \smile J_{D S M q_{\sigma}} \smile J_{D A E \sigma} .
$$

In summary, training the denoising autoencoder defined in section 2 is equivalent to performing score matching (explicit or implicit) with the energy function of Eq. 12 on Parzen density estimate $q_{\sigma}$. Such a training would typically use stochastic gradient descent, whereby samples from $q_{\sigma}$ are obtained by corrupting samples from $D_{n}$. And it may be carried out with any of these four optimization objective formulations ${ }^{5}$.

We introduced the kernel-smoothed empirical distribution $q_{\sigma}$ to show a connection between score matching and a simple denoising autoencoder. Interestingly, the regularized score matching criterion $J_{I S M r e g}(E q .6)$ that Kingma and LeCun (2010) recently introduced with the very different motivation of curing possible instabilities, was derived by approximating ${ }^{6}$ what amounts to $J_{I S M q_{\sigma}}$. From this perspective our four $q_{\sigma}$-based criteria in Eq. 15, including the denoising autoencoder, may be seen as alternative approximation-free forms of regularized score matching. A key difference is that, as is done with denoising autoencoder training, we would optimize stochastic versions of these approximation-free regularized criteria by corrupting training examples (i.e. sampling form $q_{\sigma}$ ), whereas Kingma and LeCun (2010) optimize an approximation of $J_{I S M q_{\sigma}}$, centered on the training samples only (i.e. sampling from $q_{0}$ ). Also, whereas $J_{I S M r e g}$, like the other ISM criteria, requires computing second derivatives, the stochastic version of our novel $J_{D S M q_{\sigma}}$ criterion does not, and thus appears much simpler to implement.

Note that the energy function in Eq. 12 is particular in that its scaling, which we may call its temperature, is chosen to match the corrupting noise level $\sigma^{2}$. This is required only to establish the last equivalence with the specific DAE we considered. But regarding the generic objectives $J_{I S M q_{\sigma}} \smile J_{E S M q_{\sigma}} \smile J_{D S M q_{\sigma}}$, their $\sigma$ may in principle be chosen irrespective of the form or temperature of whatever energy function is to be learnt. Interestingly, the energy function in Eq. 12, that we designed to yield the equivalence with our denoising autoencoder objective, happens to be very similar to the free energy of a Restricted Boltzmann Machine with binary hidden units and Gaussian visible units (Welling et al., 2005; Bengio et al., 2007; Swersky, 2010). The major difference is that this latter free energy does not have a global temperature scaling of the whole expression ${ }^{7}$. We designed Eq. 12 to exactly yield the denoising version of the classic autoencoder described in Section 2. But with tied weights, it may be preferable to allow for an extra positive scaling parameter $\alpha$ for the reconstruction, so that there at least exists an equivalent reparametrization of the model for scaled input

\footnotetext{
${ }^{5}$ Note however that while these $q_{\sigma}$-based objectives are formally equivalent, their stochastic gradient descent optimization, based on sampling a limited number of corrupted examples, is likely to behave differently for each objective.

${ }^{6}$ A first order Taylor expansion and a diagonal Hessian approximation are used.

${ }^{7}$ Specifically, in the free energy of a Gaussian-binary RBM, the softplus terms are not divided by $\sigma^{2}$ nor scaled in any way.
} 
data $^{8}$. This is easily obtained in the energy function by multiplying the sum of softplus terms in Eq. 12 by $\alpha$. We may even allow an arbitrary rescaling factor $\alpha_{j}$ for each hidden layer dimension independently by multiplying each softplus term by its own rescaling parameter $\alpha_{j}$, which yields the following more flexible energy function:

$$
E(\mathbf{x} ; \underbrace{\mathbf{W}, \mathbf{b}, \mathbf{c}, \alpha, \sigma_{m}}_{\theta})=-\frac{1}{\sigma_{m}^{2}}\left(\langle\mathbf{c}, \mathbf{x}\rangle-\frac{1}{2}\|\mathbf{x}\|^{2}+\sum_{j=1}^{d_{h}} \alpha_{j} \operatorname{softplus}\left(\left\langle\mathbf{W}_{j}, \mathbf{x}\right\rangle+\mathbf{b}_{j}\right)\right) .
$$

Here we have also included, as model parameter, a $\sigma_{m}$ (where $m$ stands for model) distinct from the noise $\sigma$ of the training objective ${ }^{9}$.

Our $q_{\sigma}$-based objectives $J_{I S M q_{\sigma}}, J_{E S M q_{\sigma}}$, or $J_{D S M q_{\sigma}}$ can be used as alternatives to the finite sample objective $J_{I S M q_{0}}$ (Eq. 4) advocated in Hyvärinen (2005) for learning unnormalized densities. Note that $J_{I S M q_{0}}$ is a special case of $J_{I S M q_{\sigma}}$ obtained in the limit of $\sigma \rightarrow 0$. Also, since Kingma and LeCun (2010) showed that it may be preferable to use a regularized criterion (that they derived form smoothed empirical distribution $q_{\sigma}$ ), it is likely that our $q_{\sigma}$-based criteria may, for $\sigma>0$, yield better generalization performance than the $J_{I S M q_{0}}$ advocated in Hyvärinen $(2005)^{10}$. It seems that $\sigma$ could allow one to choose an optimal bias-variance tradeoff for the finite-sample estimation of the true score matching gradient of interest $\nabla_{\theta} J_{E S M q}=\nabla_{\theta} J_{I S M q}$. While $\nabla_{\theta} J_{I S M q_{0}}$ is an unbiased estimator of it, $\nabla_{\theta} J_{I S M q_{\sigma}}=\nabla_{\theta} J_{E S M q_{\sigma}}=\nabla_{\theta} J_{D S M q_{\sigma}}$ will generally be biased when $\sigma>0$ but are also likely to have a lower variance.

Among the three equivalent score matching objectives based on $q_{\sigma}$, objective $J_{D S M q_{\sigma}}$ appears particularly interesting as a novel alternative formulation. It was motivated by both the SM principle and the DAE principle. From DAE it borrows the idea of learning to denoise artificially corrupted samples, and from SM it borrows the idea of learning a score function derived from an unnormalized density. $J_{D S M q_{\sigma}}$ may prove simpler and more efficient in practice than the mathematically equivalent $J_{I S M q_{\sigma}}$, as it does not require computing second derivatives.

Our result is also a significant advance for DAEs. First, we have defined a proper energy function for the considered DAE through Eq. 12. This will enable many previously impossible or ill-defined operations on a trained DAE, for example deciding which is the more likely among several inputs, or sampling from a trained DAE using Hybrid Monte-Carlo (Duane et al., 1987). Second, whereas using the same weight matrix ("tied weights") for the encoder and decoder is justified for RBMs, the encoderdecoder framework does not constrain that choice. Previous work on DAEs (Vincent et al., 2008; Erhan et al., 2010; Vincent et al., 2010) explored both options, often finding tied weights to yield better empirical results. Within the SM framework presented here, using tied weights between encoder and decoder now has a proper justification,

\footnotetext{
${ }^{8}$ If for example one multiplies the input values by 100 , one can obtain the same hidden representation as before by dividing $\mathbf{W}$ by 100 . But because of the tied weights this means that the reconstruction would also be divided by 100 (i.e. there is no equivalent reparametrization), unless it can be compensated by an additional scaling of the reconstruction by a parameter $\alpha$.

${ }^{9} \mathrm{We}$ would however have to set $\sigma_{m}=\sigma$ to recover a recognizable denoising autoencoder objective.

${ }^{10}$ It is also noteworthy that the experimental results of Vincent et al. $(2008,2010)$ on DAE showed that the best models, judged by their ability to extract useful features, were obtained for non negligible values of the noise parameters. Moreover this way of controlling the model's capacity worked much better than either reducing the hidden layer size or than traditional weight decay.
} 
since it follows naturally from differentiating the energy. Third, this framework opens the door to new variants that would naturally fall out from other choices of the energy function.

\section{Acknowledgements}

We would like to thank Yoshua Bengio, Olivier Delalleau and the other members of the Lisa Lab who provided timely feedback, as well as two anonymous referees whose thoughtful comments and suggestions helped improve the manuscript. This research was supported by NSERC, MITACS and FQRNT.

\section{References}

Bengio, Y., Lamblin, P., Popovici, D., and Larochelle, H. (2007). Greedy layer-wise training of deep networks. In B. Schölkopf, J. Platt, and T. Hoffman, editors, Advances in Neural Information Processing Systems 19 (NIPS'06), pages 153-160. MIT Press.

Duane, S., Kennedy, A., Pendleton, B., and Roweth, D. (1987). Hybrid Monte Carlo. Phys. Lett. B, 195, 216-222.

Erhan, D., Bengio, Y., Courville, A., Manzagol, P.-A., Vincent, P., and Bengio, S. (2010). Why does unsupervised pre-training help deep learning? Journal of Machine Learning Research, 11, 625-660.

Gallinari, P., LeCun, Y., Thiria, S., and Fogelman-Soulie, F. (1987). Memoires associatives distribuees. In Proceedings of COGNITIVA 87, Paris, La Villette.

Hinton, G. E. (2002). Training products of experts by minimizing contrastive divergence. Neural Computation, 14, 1771-1800.

Hinton, G. E., Osindero, S., and Teh, Y.-W. (2006). A fast learning algorithm for deep belief nets. Neural Computation, 18, 1527-1554.

Hyvärinen, A. (2005). Estimation of non-normalized statistical models using score matching. Journal of Machine Learning Research, 6, 695-709.

Hyvärinen, A. (2007a). Connections between score matching, contrastive divergence, and pseudolikelihood for continuous-valued variables. IEEE Transactions on Neural Networks, 18, 1529-1531.

Hyvärinen, A. (2007b). Some extensions of score matching. Computational Statistics and Data Analysis, 51, 2499-2512.

Hyvärinen, A. (2008). Optimal approximation of signal priors. Neural Computation, 20(12), 3087-3110. 
Kingma, D. and LeCun, Y. (2010). Regularized estimation of image statistics by score matching. In J. Lafferty, C. K. I. Williams, J. Shawe-Taylor, R. Zemel, and A. Culotta, editors, Advances in Neural Information Processing Systems 23 (NIPS'10), pages 1126-1134. MIT Press.

LeCun, Y. (1987). Modèles connexionistes de l'apprentissage. Ph.D. thesis, Université de Paris VI.

Lyu, S. (2009). Interpretation and generalization of score matching. In Proceedings of the 25th Conference in Uncertainty in Artificial Intelligence (UAI'09).

Marlin, B., Swersky, K., Chen, B., and de Freitas, N. (2009). Inductive principles for restricted boltzmann machine learning. In Proceedings of The Thirteenth International Conference on Artificial Intelligence and Statistics (AISTATS'10), volume 9, pages 509-516.

Movellan, J. R. (2008). Contrastive divergence in gaussian diffusions. Neural Computation, 20(9), 2238-2252.

Seung, S. H. (1998). Learning continuous attractors in recurrent networks. In M. Jordan, M. Kearns, and S. Solla, editors, Advances in Neural Information Processing Systems 10 (NIPS'97), pages 654-660. MIT Press.

Sohl-Dickstein, J., Battaglino, P., and DeWeese, M. R. (2009). Minimum probability flow learning. Technical report, arXiv:0906.4779.

Swersky, K. (2010). Inductive Principles for Learning Restricted Boltzmann Machines. Master's thesis, University of British Columbia.

Vincent, P., Larochelle, H., Bengio, Y., and Manzagol, P.-A. (2008). Extracting and composing robust features with denoising autoencoders. In W. W. Cohen, A. McCallum, and S. T. Roweis, editors, ICML'08, pages 1096-1103. ACM.

Vincent, P., Larochelle, H., Lajoie, I., Bengio, Y., and Manzagol, P.-A. (2010). Stacked denoising autoencoders: learning useful representations in a deep network with a local denoising criterion. Journal of Machine Learning Research, 11, 3371-3408.

Welling, M. and Hinton, G. E. (2002). A new learning algorithm for mean field Boltzmann machines. In ICANN '02: Proceedings of the International Conference on Artificial Neural Networks, pages 351-357, London, UK. Springer-Verlag.

Welling, M., Rosen-Zvi, M., and Hinton, G. E. (2005). Exponential family harmoniums with an application to information retrieval. In L. Saul, Y. Weiss, and L. Bottou, editors, Advances in Neural Information Processing Systems 17 (NIPS'04), pages 1481-1488, Cambridge, MA. MIT Press. 


\section{Appendix}

Proof that $J_{E S M q_{\sigma}} \smile J_{D S M q_{\sigma}}(11)$

The explicit score matching criterion using the Parzen density estimator is defined in Eq. 7 as

$$
J_{E S M q_{\sigma}}(\theta)=\mathbb{E}_{q_{\sigma}(\tilde{\mathbf{x}})}\left[\frac{1}{2}\left\|\psi(\tilde{\mathbf{x}} ; \theta)-\frac{\partial \log q_{\sigma}(\tilde{\mathbf{x}})}{\partial \tilde{\mathbf{x}}}\right\|^{2}\right]
$$

which we can develop as

$$
J_{E S M q_{\sigma}}(\theta)=\mathbb{E}_{q_{\sigma}(\tilde{\mathbf{x}})}\left[\frac{1}{2}\|\psi(\tilde{\mathbf{x}} ; \theta)\|^{2}\right]-S(\theta)+C_{2}
$$

where $C_{2}=\mathbb{E}_{q_{\sigma}(\tilde{\mathbf{x}})}\left[\frac{1}{2}\left\|\frac{\partial \log q_{\sigma}(\tilde{\mathbf{x}})}{\partial \tilde{\mathbf{x}}}\right\|^{2}\right]$ is a constant that does not depend on $\theta$, and

$$
\begin{aligned}
S(\theta) & =\mathbb{E}_{q_{\sigma}(\tilde{\mathbf{x}})}\left[\left\langle\psi(\tilde{\mathbf{x}} ; \theta), \frac{\partial \log q_{\sigma}(\tilde{\mathbf{x}})}{\partial \tilde{\mathbf{x}}}\right\rangle\right] \\
& =\int_{\tilde{\mathbf{x}}} q_{\sigma}(\tilde{\mathbf{x}})\left\langle\psi(\tilde{\mathbf{x}} ; \theta), \frac{\partial \log q_{\sigma}(\tilde{\mathbf{x}})}{\partial \tilde{\mathbf{x}}}\right\rangle d \tilde{\mathbf{x}} \\
& =\int_{\tilde{\mathbf{x}}} q_{\sigma}(\tilde{\mathbf{x}})\left\langle\psi(\tilde{\mathbf{x}} ; \theta), \frac{\frac{\partial}{\partial \tilde{\mathbf{x}}} q_{\sigma}(\tilde{\mathbf{x}})}{q_{\sigma}(\tilde{\mathbf{x}})}\right\rangle d \tilde{\mathbf{x}} \\
& =\int_{\tilde{\tilde{\mathbf{x}}}}\left\langle\psi(\tilde{\mathbf{x}} ; \theta), \frac{\partial}{\partial \tilde{\mathbf{x}}} q_{\sigma}(\tilde{\mathbf{x}})\right\rangle d \tilde{\mathbf{x}} \\
& =\int_{\tilde{\mathbf{x}}}\left\langle\psi(\tilde{\mathbf{x}} ; \theta), \frac{\partial}{\partial \tilde{\mathbf{x}}} \int_{\mathbf{x}} q_{0}(\mathbf{x}) q_{\sigma}(\tilde{\mathbf{x}} \mid \mathbf{x}) d \mathbf{x}\right\rangle d \tilde{\mathbf{x}} \\
& =\int_{\tilde{\mathbf{x}}}\left\langle\psi(\tilde{\mathbf{x}} ; \theta), \int_{\mathbf{x}} q_{0}(\mathbf{x}) \frac{\partial q_{\sigma}(\tilde{\mathbf{x}} \mid \mathbf{x})}{\partial \tilde{\mathbf{x}}} d \mathbf{x}\right\rangle d \tilde{\mathbf{x}} \\
& =\int_{\tilde{\mathbf{x}}}\left\langle\psi(\tilde{\mathbf{x}} ; \theta), \int_{\mathbf{x}} q_{0}(\mathbf{x}) q_{\sigma}(\tilde{\mathbf{x}} \mid \mathbf{x}) \frac{\partial \log q_{\sigma}(\tilde{\mathbf{x}} \mid \mathbf{x})}{\partial \tilde{\mathbf{x}}} d \mathbf{x}\right\rangle d \tilde{\mathbf{x}} \\
& =\int_{\tilde{\mathbf{x}}} \int_{\mathbf{x}} q_{0}(\mathbf{x}) q_{\sigma}(\tilde{\mathbf{x}} \mid \mathbf{x})\left\langle\psi(\tilde{\mathbf{x}} ; \theta), \frac{\partial \log q_{\sigma}(\tilde{\mathbf{x}} \mid \mathbf{x})}{\partial \tilde{\mathbf{x}}}\right\rangle d \mathbf{x} d \tilde{\mathbf{x}} \\
& =\int_{\tilde{\mathbf{x}}} \int_{\mathbf{x}} q_{\sigma}(\tilde{\mathbf{x}}, \mathbf{x})\left\langle\psi(\tilde{\mathbf{x}} ; \theta), \frac{\partial \log q_{\sigma}(\tilde{\mathbf{x}} \mid \mathbf{x})}{\partial \tilde{\mathbf{x}}}\right\rangle d \mathbf{x} d \tilde{\mathbf{x}} \\
& =\mathbb{E}_{q_{\sigma}(\tilde{\mathbf{x}}, \mathbf{x})}\left[\left\langle\psi(\tilde{\mathbf{x}} ; \theta), \frac{\partial \log q_{\sigma}(\tilde{\mathbf{x}} \mid \mathbf{x})}{\partial \tilde{\mathbf{x}}}\right\rangle\right]
\end{aligned}
$$

Substituting this expression for $S(\theta)$ in Eq. 16 yields

$$
\begin{aligned}
J_{E S M q_{\sigma}}(\theta)= & \mathbb{E}_{q_{\sigma}(\tilde{\mathbf{x}})}\left[\frac{1}{2}\|\psi(\tilde{\mathbf{x}} ; \theta)\|^{2}\right] \\
& -\mathbb{E}_{q_{\sigma}(\mathbf{x}, \tilde{\mathbf{x}})}\left[\left\langle\psi(\tilde{\mathbf{x}} ; \theta), \frac{\partial \log q_{\sigma}(\tilde{\mathbf{x}} \mid \mathbf{x})}{\partial \tilde{\mathbf{x}}}\right\rangle\right]+C_{2} .
\end{aligned}
$$


We also have defined in Eq. 9,

$$
J_{D S M q_{\sigma}}(\theta)=\mathbb{E}_{q_{\sigma}(\mathbf{x}, \tilde{\mathbf{x}})}\left[\frac{1}{2}\left\|\psi(\tilde{\mathbf{x}} ; \theta)-\frac{\partial \log q_{\sigma}(\tilde{\mathbf{x}} \mid \mathbf{x})}{\partial \tilde{\mathbf{x}}}\right\|^{2}\right]
$$

which we can develop as

$$
\begin{aligned}
J_{D S M q_{\sigma}}(\theta)= & \mathbb{E}_{q_{\sigma}(\tilde{\mathbf{x}})}\left[\frac{1}{2}\|\psi(\tilde{\mathbf{x}} ; \theta)\|^{2}\right] \\
& -\mathbb{E}_{q_{\sigma}(\mathbf{x}, \tilde{\mathbf{x}})}\left[\left\langle\psi(\tilde{\mathbf{x}} ; \theta), \frac{\partial \log q_{\sigma}(\tilde{\mathbf{x}} \mid \mathbf{x})}{\partial \tilde{\mathbf{x}}}\right\rangle\right]+C_{3}
\end{aligned}
$$

where $C_{3}=\mathbb{E}_{q_{\sigma}(\mathbf{x}, \tilde{\mathbf{x}})}\left[\frac{1}{2}\left\|\frac{\partial \log q_{\sigma}(\tilde{\mathbf{x}} \mid \mathbf{x})}{\partial \tilde{\mathbf{x}}}\right\|^{2}\right]$ is a constant that does not depend on $\theta$.

Looking at equations 17 and 18 we see that $J_{E S M q_{\sigma}}(\theta)=J_{D S M q_{\sigma}}(\theta)+C_{2}-C_{3}$. We have thus shown that the two optimization objectives are equivalent. 